\title{
Nitrate-selective gallium nitride transistor-based ion sensors with low detection limit
}

\author{
M. Myers ${ }^{1,2}$, A. Podolska', T. Pope ${ }^{2}$, F.M.L. Khir', U.K. Mishra ${ }^{3}$, B.D. Nener', M.V. Baker', G. Parish ${ }^{2}$ \\ ${ }^{1}$ CSIRO Earth Science and Resource Engineering, Australia \\ ${ }^{2}$ The University of Western Australia, 35 Stirling Hwy, Crawley WA 6009, Australia \\ ${ }^{3}$ University of California, Santa Barbara CA 93106, USA \\ M018, 35 Stirling Hwy, Crawley, WA 6009, Australia \\ giacinta.parish@uwa.edu.au
}

\begin{abstract}
:
AIGaN/GaN heterostructures functionalised with ion-selective membranes have been used to form field effect transistor (FET)-like nitrate sensors which operate without the requirement for a reference electrode. These sensors demonstrate a wide range of sensitivity of $1 \times 10^{-6}-1 \times 10^{-2} \mathrm{M}(62 \mathrm{ppb}-$ $620 \mathrm{ppm})$ and a low detection limit below $1 \times 10^{-7} \mathrm{M}(6.2 \mathrm{ppb})$. The low detection limit for this device is superior to the original published results for standard ion selective electrodes (ISEs) using a similar membrane chemistry. The membrane chemistry for these sensors was modified from the original recipe in order to optimise application to small area devices.
\end{abstract}

Key words: $\mathrm{AlGaN} / \mathrm{GaN}$, nitrate sensor, transistor, ion-selective membrane

\section{Intrroduction}

Ion-sensitive field effect transistors (ISFETs) are a solid-state solution to potentiometric sensing that offer the advantages of compact size, mass-producibility, on-chip integration, speed, and sensitivity [1]. To date the majority of investigations use Si-based ISFETs. While they have shown promise, the gate dielectrics are highly sensitive to charging effects and can be chemically unstable in aqueous solutions, and the devices require a reference electrode to bias the gate of the transistor beyond threshold (so as to allow majority carriers to travel through the conductive channel) [2]. Also, Si itself is not chemically or thermally robust.

AIGaN/GaN high electron mobility transistors (HEMTs), which have recently come to dominate the high-power electronics market, have the unique benefits of high sensitivity of device conductivity to surface charge, fast response, and excellent chemical and thermal stability [1]. AlGaN and $\mathrm{GaN}$ are members of the III-nitride alloy family, which has become extremely technologically important for a variety of applications such as wireless base stations, solid-state lighting, automotive electronics, power conditioning and information storage. The properties of AIGaN/GaN HEMTs are also very attractive for sensors. Importantly, in contrast to Si-based transistors, AIGaN/GaN HEMTs do not require a gate bias to be turned on; when used as ion sensing transistor devices no reference electrode is therefore required for aqueous operation [3,4], as shown in Fig. 1. This is a significant advantage over electrochemical cell techniques.

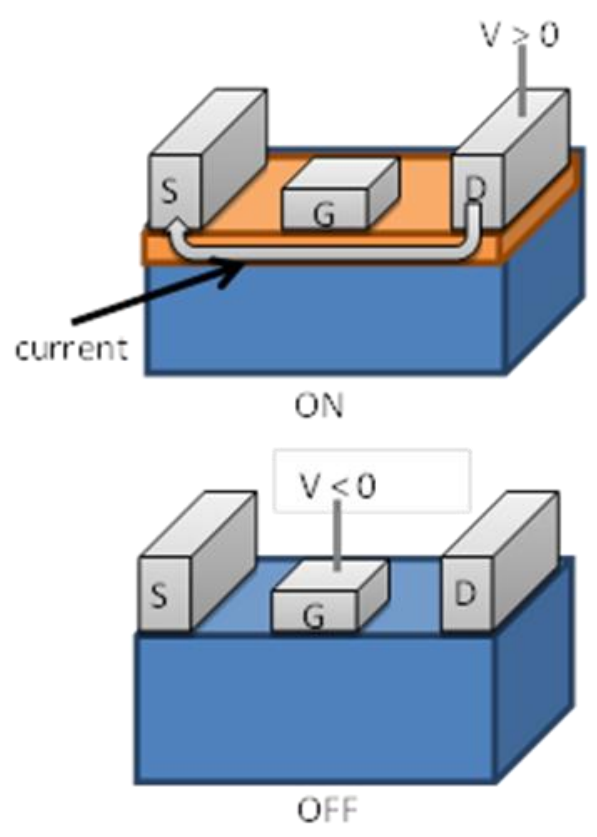

Fig. 1 Schematic of AlGaN/GaN HEMT showing ON and OFF operation. In all transistors positive current flows source (S) to drain (D) when device is $O N$ and a positive voltage is applied to the gate $(G)$. However, unlike silicon-based devices, the channel is turned OFF by application of a negative gate voltage. 
AlGaN/GaN HEMTs have gained recent attention for a variety of sensor applications [1, 5-9]. Group III-nitrides (GaN, AIN and their alloys) have high mechanical, thermal, and chemical stability. A particular advantage of the $\mathrm{AlGaN} / \mathrm{GaN}$ heterostructure is the formation of a 2D electron gas sheet (2DEG) close to the surface, which forms the channel of the transistor. Due to the exponential relationship between charge changes on the the surface (gate) of the device and the 2DEG channel charge, not only can the devices directly sense charged particles adsorbed onto the exposed gate area, but the measured source-drain current is an amplification of these charge changes. By functionalising the transistor gate to be selective, a wide variety of sensors may be fabricated, without the need for susceptible dielectrics upon which Si devices rely. To date, AlGaN/GaN-based chemical sensors have been primarily investigated for determining $\mathrm{pH}$ and detecting gases $[6,10,11]$ and polar species (including ammonium [5] and polar liquids [12]). AIGaN/GaN based biosensors have focused on biomedical applications including cancer and disease detection and health monitoring [7]. In this paper we present the first results on AIGaN/GaN nitrate sensors using ion-selective membranes.

\section{Experiment}

Fig. 2a shows a schematic cross-section of a HEMT based sensor with an ion-sensitive membrane on the gate area. Ungated devices with 4-point contact geometry were fabricated on standard AIGaN/GaN FET wafers with $2 \mathrm{~nm}$ GaN cap.Fig.2b shows the device layout.
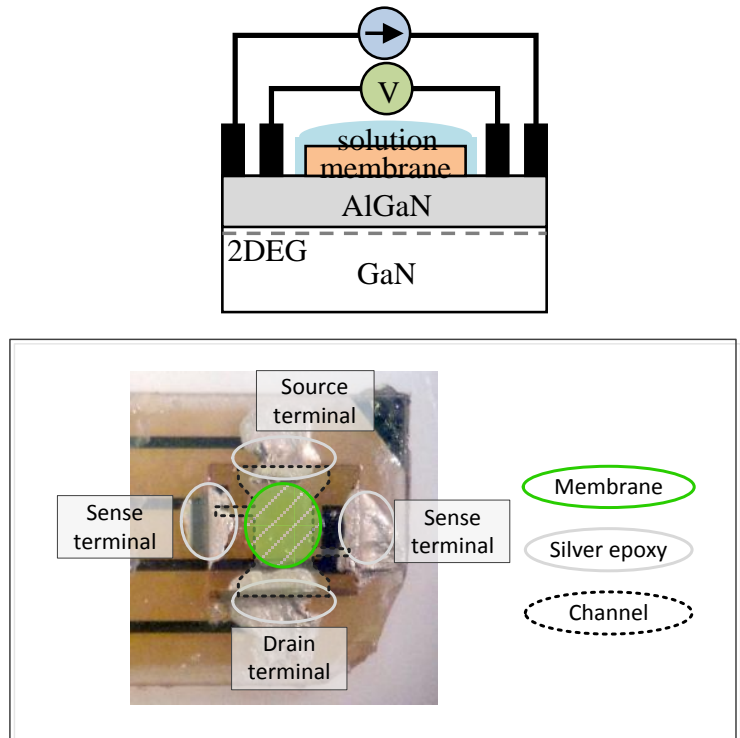

Fig.2 a) Above: Schematic cross-section of AlGaN/GaN HEMT-based sensor with ion-sensitive membrane on the gate. b) Below: Top view of one of the devices used for experiments.
A plasticized PVC-based membrane containing a nitrate selective ionophore was formed in situ by dropping the solution directly onto the gate region. The membrane formulation $(1 \mathrm{mg}$ of tetradodecylammonium nitrate, $66 \mathrm{mg}$ of 2nitrophenyloctyl ether and $33 \mathrm{mg}$ of high molecular weight polyvinyl chloride dissolved in THF) was adapted from a similar formulation optimised for traditional ISEs [13. During measurements a $100 \mu \mathrm{A}$ constant current source was connected to drain and source terminals, and voltage drop across the channel measured viatwo voltage sense terminals.

\section{Results}

The results in Fig. 3 show the sensor responds to aqueous nitrate ions over a wide range of concentrations. This particular device shows a working range of $1 \times 10^{-6}-1 \times 10^{-2} \mathrm{M}(62 \mathrm{ppb}-$ $620 \mathrm{ppm}$ ) (higher concentrations were not tested) and lower detection limit below $1 \times 10^{-7} \mathrm{M}$ (6.2 ppb). Importantly, the detection limit for this device is superior to the original published results for standard ISEs using the same membrane [13].

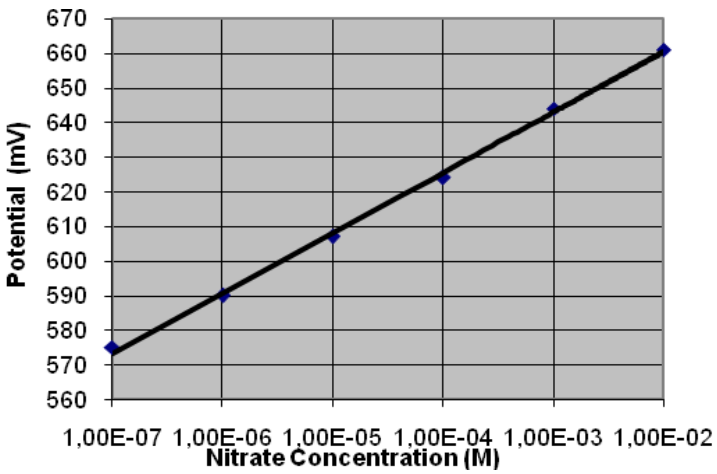

Fig. 3. Example of results for an AIGaN/GaN HEMT device with nitrate selective membrane, tested over a range of concentrations $\left(1 \times 10^{-7} \mathrm{M}-1 \times 10^{-2} \mathrm{M} ; 6.2 \mathrm{ppb}\right.$ - 620 ppm)

\section{Conclusions}

This work has demonstrated that the sensor under development is able to effectively detect nitrate ions without the use of a reference electrode. Other general ion sensitivity measurements on a series of different (but unfunctionalised) devices have shown that sensitivity can be increased by orders of magnitude with improved design, thus even lower detection limits are expected through optimisation of the AIGaN/ GaN device design. Overall the device has the potential to be an effective and compact sensor with the advantages of high chemical, mechanical and thermal stability associated with $\mathrm{AlGaN} / \mathrm{GaN}$ FETs. 


\section{Acknowledgements}

Partial funding by the Australian Research Council DP0988241 and scientific and technical assistance of the WA Node of the Australian National Fabrication Facility.

\section{References}

[1] G. Steinhoff et al, Advanced Functional Materials, 13, 841-845 (2003); doi: 10.1002/adfm. 200304397

[2] P. Bergveld, Thirty years of ISFETOLOGY - What happened in the past 30 years and what may happen in the next 30 years, Sensors and Actuators B - Chemical, 88, 1-20 (2003); doi: 10.1016/S0925-4005(02)00301-5

[3] A. B. Encabo et al., Ultrathin GaN/AIN/GaN solution-gate field effect transistor with enhanced resolution at low source-gate voltage,Sensors and Actuators B - Chemical, 142, 304-307 (2009); doi:10.1016/jsnb.200907016,

[4] A. Podolska et al., lon versus $\mathrm{pH}$ sensitivity of ungated $\mathrm{AlGaN} / \mathrm{GaN}$ heterostructure-based devices,Applied Physics Letters, 97,012108(2010); doi: 10.1063/1.3462323

[5] Y. Alifragis et al,AlGaN/GaN high electron mobility transistor sensor sensitive to ammonium ions, Physica Status Solidi A 204, 2059-2063 (2007); doi: 10.1002/pssa.200674885

[6] M. Stutzmann, J.A. Garrido, M. Eickhoff, Twodimensional charge carrier systems for chemical sensors: AlGaN/GaN and diamond,Proceedings of IEEE Sensors 2003, 1153-1158(2003); doi: 10.1109/ICSENS.2003.1279125
[7] B.S. Kang, H.T. Wang, F. Ren, S.J. Pearton,Electrical detection of biomaterials using. AlGaN/GaN high electron mobility transistors, Journal of Applied Physics 104, 031101 (2008); doi:10.1063/1.2959429

[8] M.Hofstetter et al.,Development and evaluation of gallium nitride-based thin films for $x$-ray dosimetry, Physics in Medicine and Biology 56, 3215-3231 (2011); doi:10.1088/00319155/56/11/004

[9] S. Schwarz et al., DNA-sensor based on AlGaN/GaN high electron mobility transistor, Physica Status Solidi A 208, 1626-1629 (2011); doi: 10.1002/pssa.201001041

[10] Chen et al., Improved hydrogen-sensing performance of a $\mathrm{Pd} / \mathrm{GaN}$ Schottky diode with a surface plasma treatment approach, Sensors and Actuators B-Chemical159,159-62 (2011); doi: 10.1016/jsnb.201106066

[11] Higuchi et al., Field effect hydrogen sensor device with simple structure based on GaN,Sensors and Actuators B-Chemical140, 7985(2009); doi: 10.1016/jsnb.200904031

[12] Kokawa et al., Liquid-phase sensors using opengate $\mathrm{AIGaN} / \mathrm{GaN}$ high electron mobility transistor structure, Journal of Vacuum Science \& Technology $B, \quad 24, \quad 1972-76 \quad$ (2006); doi: 10.1116/1.2214701

[13] S.S.M. Hassan, H.E.M. Sayour, S.S. AlMehrezi,Analytica Chimica Acta 581, 13-18 (2007); doi: 10.1016/j.aca.2006.08.011 